\title{
Nanorobotics in Drug Delivery Systems for Treatment of Cancer: A Review
}

Glécia Virgolino da Silva Luz ${ }^{1,5^{*}}$, Kleber Vânio Gomes Barros ${ }^{1,2,5}$, Fábio Vladimir Calixto de Araújo ${ }^{1,5}$, Gabriela Barbosa da Silva ${ }^{3,5}$, Pedro Augusto Ferreira da Silva ${ }^{3,5}$, Roxana Claudia Iquize Condori ${ }^{4,5}$ and Lourdes Mattos Brasil $^{1,5}$

1. Post-Graduate Program in Biomedical Engineering, Campus Gama-FGA, University of Brasília-UnB, Brasília, 72444-240, Brazil

2. Pharmaceutical Technology and Quality Control of Medicines, University Center of Distrito Federal-UDF, Brasília, 70390-045, Brazil

3. University of Brasília-UnB/ UnB at Gama-FGA, Brasília, 72444-240, Brazil

4. Post-Graduate Program in Health Sciences, Campus Darcy Ribeiro, University of Brasília-UnB, Brasília, 70910-900, Brazil

5. Nanotechnology Laboratory - University of Brasília at Gama (NANOTEC-FGA/UnB), Brasília, 72444-240, Brazil

\begin{abstract}
This review aims at giving an overview of the present status of nanorobotics in cancer therapy. With the aid of biotechnology, molecular biology (as engineered organism) and molecular medicine can develop fully self-sufficient nanorobots/nanobots. The nanorobotics considered, as a wonderful vision of medicine in the future are an advanced submicron device generally made of bio-nanocomponents. It has an eminence future in the drug delivery technology target in cancer, the disease that leading cause of death among younger than 85 years. Nanorobots could carry and deliver large amounts of anti-cancer drugs into cancerous cells without harming healthy cells, reducing the side effects related to current therapies like damage of the conventional chemotherapy. This paper presents a study on different approaches employed towards cancer treatment using nanorobots. Further, it also provides an insight into the future scope in this field of study.
\end{abstract}

Key words: Nanorobotics, cancer therapy, application of nanorobots, nanorobotics for cancer.

\section{Nomenclature}

CMOS: Complementary metal oxide semiconductor

CNS: Central nervous system

CNT: Carbon nanotubes

$\mathrm{CO}_{2}$ : Carbon dioxide gas

DDS: Drug delivery system

DNA: Deoxyribonucleic acid

HA: Hydroxyapatite

HD: Hodgkin's disease

IMS: Institute for healthcare informatics

MEMS: Microelectro mechanical systems

NEMS: Nanoelectro mechanical Systems

NOS: Nitric oxide synthase

$\mathrm{O}_{2}$ : $\quad$ Oxygen gas

*Corresponding author: Glécia Virgolino da Silva Luz, collaborating professor of PostGraduation Program in Biomedical Engineering at Campus Gama-UnB, research fields: material science, nanotechnology and nanopowders.

\author{
RBCs: Red blood cells \\ SPMs: Scanning probe microscopes \\ SPIONs: Superparamagnetic nanoparticles of iron oxide \\ SWNTs: Single-walled carbon nanotubes \\ Tax: Paclitaxel
}

\section{Introduction}

The nanotechnology, an impressive technological tendency has been highlighted by researchers in recent decades and it goes through the rapid growth of electronics for applications in communication, health care (called nanomedicine) and environmental monitoring. Much research is currently focused on the scientific bottlenecks that pervade the longevity of living organisms, especially human beings. Among these bottlenecks are principally diseases having little or no alternative treatment and healing. An alternative 
diagnostic and/or treatment that have been displayed in the academic community is called DDS (Drug delivery system) [1]. As said by Abhilash (2010) [2], and confirmed with the sequenced research, an interdisciplinary approach would ensure that the exciting potential of nanomedicine many levels would be a concreted reality. The combination of the skills of various professionals in the field of medicine, pharmaceuticals, chemistry and physics really facilitate research on nanomedicine or studies DDS e nanorobotics [2].

NEMS (Nanorobots are NanoElectroMechanical Systems), which represent a new frontier in miniaturization, near the MEMS (Microelectro mechanical systems) that constitute today a multibillion-dollar industry. Nanorobotics and more generally, NEMS research involves design (which often is biologically inspired), prototyping, fabrication, programming and applications such as biomedical nanotechnology. Robotics at any scale involves sensing, control, actuation and propulsion, power, communications, interfacing, and programming and coordination. Emphasis on actuation, which is a fundamental requirement for robotics.

A major obstacle facing this technology nowadays is to lack effective processes for building the nanoscale structures required by the envisaged applications. Research at the University of Southern California, Los Angeles, Laboratory for Molecular Robotics and elsewhere shows that nanomanipulation with SPMs (Scanning probe microscopes) provides an effective approach for constructing nanostructures from the bottom up, by assembling building blocks that result from chemical synthesis (in general, molecules or colloidal nanoparticles). The primary shortcoming of this approach is its sequential nature and the associated low throughput. High throughput may be achieved, however, by massively parallel assembly operations using SPM multichip arrays, which are built by MEMS techniques. For example, an IBM group is building multichip for digital storage applications that are expected to achieve densities on the order of a few $\mathrm{Tb} / \mathrm{in}^{2}$ [3].

Nanorobots have dimensions close those of biological cells and organelles. This open a colossal array of potential applications in environmental watching for microorganisms and in health care as an example, imagine artificial cells (nanorobots) that patrol the cardiovascular system, notice tiny concentrations of pathogens, and destroy them. This might quantity to a programmable system, and may need sweeping implications in medication, inflicting a paradigm shift from treatment to bar. Alternative applications like cell repair can be attainable if nanorobots were sufficiently small to penetrate the cells. Additionally, minuscule sensors and actuators square measure required if the rising vision of a physically coupled ascended info infrastructure.

Conforming to the Global Oncology Trend Report, by the Quintiles IMS Institute, global spending on cancer medications has reached to $\$ 100$ billion in 2014 [4]. Cancer treatment is probably the main reason for the development of nanorobotics. Chemotherapeutic agents used in cancer square measure distributed nonspecifically within the body wherever they have an effect on each cancerous and traditional cells, thereby limiting the dose doable inside the growth and additionally leading to suboptimal treatment as a result of excessive toxicities. Molecularly targeted medical care has emerged in concert approach to beat the shortage of specificity of standard therapy agents against cancer [5]. Nanoparticles, by exploitation each passive and active targeting strategies, will enhance the intracellular concentration of medicine in cancer cells whereas avoiding toxicity in traditional cells [6]. Thereby, this Review focuses on recent advances and technology development of nanorobotics harvesting specifically for Drug Delivery Systems application for treatment of cancer.

\section{Materials}

In the material selection was included researches 
that relate studies and technology development of nanorobotics harvesting specifically for Drug Delivery Systems application for treatment of cancer. The search strategy was, first, to look for the definitions of Nanorobots, its structure and design, in more traditional documents to most current. A search was done in the databases of PubMed, Medline, Science Direct, SCOPUS, ISI web of knowledge and grey literature as Google scholar. No restrictions of language or time were applied. The following search terms were used: "drug delivery" and "cancer" or "neoplasms" or "treatment of cancer". Also synonyms and derivate of the terms were used for finding more articles. To have a wide-ranging search and to find possible relevant articles, manual search was done on reference list of articles. They were carried brief accounts of the studies found. It was also conducted a statistical study with respect to the amount of work found in the aforementioned database.

\section{Results and Discussion}

According to the research conducted, in general, are defined nanorobots and are included reports of publications about the topics selected in searches. A brief statistical study was carried out using data of found works.

\subsection{Nanorobots/Nanobots}

Eric Dexler in 1986 was already talking about creating machines built atom by atom [7]. Currently, it is expected that nanorobots are the future of technology, health and the environment [8]. The applications of nanorobots are many.

Nanorobots are tiny machines that can work as today's machines, but more exactly, the development of this new technology, brings benefits in medicine, industry, and others areas [1]. They can be applied to solve problems of energy conversion, using catalytic nanomotors, recent studies search the ability to increase the velocity, force, lifetime, of synthetic nanomotors, some nanomotors are able to move, autonomously at speeds approaching 100 body lengths per second. The improving of velocity, motion control and the lifetime of catalytic nanomotors is very important to create power in chip microsystems powered by autonomous transports. Besides, it is possible to apply this new technology to create a laboratory on a chip device [9].

Another application is to solve infertility problems. German engineers built a nanorobot that connects to the scourge of sperm and acts as an engine by boosting the research aims to make it more accessible and effective fertility treatments [10]. Problems as pollution can also be solved with nanorobots, engineers at the University of California, for instance, are using nanorobots to capture the excess carbon dioxide in the waters of lakes, rivers and even oceans [11].

\subsection{Structure and Design of Nanorobots}

Two types of nanorobots are most widely researched, organic and inorganic. The organic nanorobots, also called bionanorobots, are manufactured using viruses and bacteria DNA cells. Such nanorobot is less toxic to the organism. Inorganic nanobots are created diamond structures [12], synthesized proteins [13] and others types of material. These types of robots are more toxic, a way of reducing this problem is to encapsulate the robot, this method also avoids its destruction by the body's defense system, this problem, has been a major challenge faced by researchers [14]. With knowledge about biological motors of living cells, scientists can learn how to power micro sized and nano-sized machines with catalytic reactions [15].

A team of South Korean scientists, of Chonnam National University, has developed organic nanorobots made with the salmonella bacteria genetically modified in order to not show toxicity, this nanorobot is attracted to molecules released by cancer cells and was named Bacteriobot, with about three micrometers. When they reach the cancer cells, they release the drug, the Bacteriobot were designed to attack colorectal tumors 
and achieved satisfactory results in tests with laboratory rats [16].

At Rice University, Texas, United States, a team has created nanomachines that resemble nanocars. The molecules have the basic mechanics of a car: wheels, made with carbon 60, chassis, comprising planar molecules and axes connecting the wheels to the chassis by triple bonds of carbon, these nanocars are able to move over a surface when supplied [16]. Another study, at the Chemistry Institute of the Federal Fluminense University, has developed a nanovalve, formed by a reservoir closed by a door, where dye molecules are stored, they can move out in a controlled manner when the door open. This device is also organic, composed of silica $\left(\mathrm{SiO}_{2}\right)$, beta-cyclodextrins and organometalic molecules, such nanovalves can be applied in Drug Delivery Systems [17].

Some research uses proteins, to feed nanomotors that can move large objects, DNA hybridization, antibody protein also is used in the construction of nanorobots. Different types of chemical substances can be employed to functionalize a nanorobot [10].

Some nanorobots are made of liquid crystal elastomers and are light-activated, presenting advantage is due to an ease internal engine and propulsion, as the one developed by researchers at the Max Planck Institute in Germany that has been made to mimic protozoa, and its composition is such that the body is is green light self-propelled [18].

One type of inorganic nanorobot was made with an array of tiny planar coils generating fields, these fields create magnetic blocks, representing a possible position for the nanorobot, and they also possess magnetic disk and a lower surface which allows sliding the positions that can be take. The prototype is about two millimeters in diameter, but the researchers plan to improve the design to further decrease the nanorobot size to about 250 micrometers in diameter [19].

\subsection{Nanorobotics and DDS}

Predictions about the use of nanorobotics considered applications in Central Nervous System (CNS) [20, 21], cancer treatment [22-25], body surveillance, delicate surgeries, endoscopy [21, 26], among others. Challenges such as limitations of nanotechnology and few studies focused on the fundamental understanding of behavior in the nanoworld, difficult handling and construction of these nanomachines [23, 27, 28].

In nanomedicine, it has been explored in DDS, which act directly on target points of the human body. Researchers develop systems able to deliver drugs in specific locations also controlling up the dosage and frequency of this release. Drug Delivery Systems can be applied in the treatment of articular diseases, dental, diabetes, cancer and other [1, 2, 29-33].

Diseases such neoplasms, hepatitis, diabetes [34], pulmonary [35, 36], dentistry [37] and cancer [23] can be used nanorobotics technology as a means of implementing the DDS. One of the advantages of this technology is the diagnosis and treatment of the diseases with minimum prejudice to the healthy cells lowering the risk of unfavorable effect [21, 38], directing healing and reconstructive treatment at the cellular and subcellular stances [34].

\subsection{Technology Applied in Nanorobotics for Use as DDS}

Recent improvements in drug delivery turns up higher quality in targeted drug delivery that identify the specific cells with the self of nanosensors and regulate the discharge by use of smart drugs [39].

Some researchers classify nanorobots in drug delivery and therapeutics according to the their applications, which are described below [39-44]:

Pharmacyte: Classified as medical nanorobots size of 1-2 $\mu \mathrm{m}$ able to carrying up $1 \mu \mathrm{m}^{3}$ a given drug in the tanks. They are controlled using mechanical systems for sorting pumps. Depending on the situation the weight is discharged in the extracellular fluid or cytosol (the aqueous component of the cytoplasm of a cell). They are provided with a molecular markers or chemo 
tactic sensors that guarantees full targeting accuracy. Glucose and oxygen extracted from the local environments such as blood, intestinal fluid and cytosol are the onboard power supply. After the nanorobot completing tasks they can be removed or recovered by centrifuge nanapheresis [39-41].

Diagnosis and Imaging: The authors cite microchips that are overlaid with human molecules. The chip is projected to send an electrical signal when the molecules detect a disease. Gives an example of special sensor nanobots that can be introduced into the blood under the skin where they verify blood contents and notify of any possible diseases. They can also be used to monitor the sugar level in the blood. Advantages are the low price to produce and easily to manipulate [39, 45].

Respirocytes: It's about an artificial red blood cell which is a blood-borne spherical $1 \mu \mathrm{m}$ diamondoid 1,000-atmosphere pressure vessel with reversible molecule-selective pumps. The power is obtained by endogenous serum glucose. This artificial cell is able to give 236 times more oxygen to the tissues per unit volume than RBCs (Red blood cells) and to administer acidity. The nanomachine is constructed with 18 billion atoms justly organized in a diamondoid pressure tank that is pumped full of up to 3 billion oxygen $\left(\mathrm{O}_{2}\right)$ and carbon dioxide $\left(\mathrm{CO}_{2}\right)$ molecules. It is possible to release these gasses from the tank. Gas concentration sensors on the outside will signal when it is time to discharge $\mathrm{O}_{2}$ and unload $\mathrm{CO}_{2}[39,40]$.

Clottocytes: This nanorobot is classified with a unique biological capability: "instant" hemostasis using clottocytes, or artificial mechanical platelets. It is known that platelets are roughly spheroidal nucleus-free blood cells measuring approximately 2 $\mu \mathrm{m}$ in diameter. Platelets join at a place of bleeding. There they are activated, becoming tacky and lumping together to form a tampon that aid stamp the blood vessel and stop the bleeding. They also delivery substances that help promote coagulating. Another interesting feature is its ability to perform phagocytosis of foreign particles and killing of microfilarial larval parasites. A complete functional design is elaborate but the work of Freitas (2016) [46] focus on the purely mechanical aspects of the hemostatic function of platelets, and report the function in a small in vivo population of medical nanorobotic devices.

Microbivores: It is an oblate spheroidal device for nanomedical applications with $3.4 \mu \mathrm{m}$ in diameter along its major axis and $2.0 \mu \mathrm{m}$ in diameter along its minor axis. Composed precisely organized by 610 billion atoms in a $12.1 \mu^{3}$ geometric volume. The nanobot can continually consume up to $200 \mathrm{pW}$. This power is used for digest trapped microbes. Microbivores have different characteristics of natural or antibiotic-assisted biological phagocytic defenses, acting as approximately up to 1,000 times faster. Another distinctive feature is related to the ability to phagocyte approximately 80 times more efficiently than macrophages agents, in terms of volume/sec digested per unit volume of phagocytic agent [42].

Thus, according to the existing technological proposals, nanorobots are an efficient and innovative way for applications in nanomedicine, including DDS and theranostic [47] (diagnostic and therapeutic). Searching keywords "drug delivery systems" in database Periódicos CAPES [48], it was obtained 176, 511 publications. Only $0.21 \%$ is related to nanorobotics, and this amount of work only $8 \%$ have the relationship "drug delivery systems and nanorobotics". Another database searched was Web Of Science [49]. The results were 113,896 publications with the keyword "drug delivery" and 201 for nanorobotics. The survey also showed that only $0.02 \%$ was published with the correlation "drug delivery and nanorobotics". Before the number of published papers, it is noted that much more should be done so that nanomedicine can grow apace with the help of nanorobotics in treating diseases, in particular cancer. 


\subsection{Drug Delivery Systems for Anticancer Drugs}

The therapeutic index of most anticancer drugs is narrow, causing toxicity to normal stem cells, hematological adverse effects, gastrointestinal among other. Doxorubicin is used in several types of cancer, such as HD (Hodgkin's Disease), in which treatment is administered in combination with other antineoplastic agents in order to reduce their toxicity [50]. The Paclitaxel is administered by intravenous infusion and plays a role in the treatment of breast cancer. Among the adverse effects encountered some serious, are bone marrow suppression and cumulative neurotoxicity [51]. Cisplatin is an alkylating agent which causes intra DNA binding filaments. Some of its side effects are nausea and severe vomiting as well as can be nephrotoxic [51]. Camptothecin is used in the treatment of neoplasias due to inhibition of type I topoisomerases, an essential enzyme for cellular replication genetic material [50]. Several efforts have been implemented with the intention of using nanotechnology to develop DDS that can minimize the harmful effects of conventional therapies. Clinical trials are studies in humans to measure the parameters of safety and efficacy of new drugs, it is essential for the arrival of new therapeutic alternatives in the market [52]. Anyway, just a few Drug Delivery Systems reached more advanced stages of clinical evaluation such as basically consisting of doxorubicin, paclitaxel, camptothecin and platinum complexes Anyway just a few DDS reached more advanced stages of clinical evaluation such as basically consisting of doxorubicin, paclitaxel, camptothecin and platinum complexes [53].

Doxorubicin was stacked on the surface of Single-Walled Carbon Nanotubes (SWNTs) [54]. The Doxorubicin was employed as polymer prodrug/collagen hybrid in metastatic tumor cells. The use of a polymeric prodrug nanotechnology applied to the treatment of neoplasia shows up as a new development in this area boundary [55]. Superparamagnetic Nanoparticles of Iron Oxide
(SPIONs) loaded with doxorubicin were coated with modified inulin and evaluated when the potential use in antineoplastic therapy [56]. The search for biocompatible materials and can serve as a Drug Delivery System is always the focus of nanotechnology. Nanoparticle HA (Hydroxyapatite) - a major constituent of bone and teeth - were used to carry Paclitaxel (Tax), an antineoplastic agent - and the results suggest good expectation with treatment starting from hydrophobic drugs [57]. Searching carbon materials nanoscale graphene oxide was tested as a drug carrier of anti-cancer [58]. Another possible application area of the drug delivery system is especially important in the intrathecal route of administration for the relief of pain related to certain types of cancer. The application Drug Delivery System Intrathecal may be useful in refractory pain to other of administration or even in cases of persistent pain [59].

Again, observing the research with the themes "Drug delivery Systems and Cancer" found a total of 31134 publications [49]. As noted in recent years, the interest increase the DDS is directly associated with the need for alternative conventional chemotherapeutics which possess some serious side effects to the patient [60].

\subsection{Limitations of Chemotherapy}

The Conventional chemotherapeutic agents work by destroying rapidly dividing cells, which is the main property of neoplastic cells. This is why chemotherapy also damages normal healthy cells that divide rapidly such as cells in the bone marrow, macrophages, digestive tract, and hair follicles [61]. The conventional chemotherapy is that it cannot give selective action only to the cancerous cells. This results in common side effects of most chemotherapeutic agents which include myelosuppression (decreased the production of white blood cells causing immunosuppression), mucositis (inflammation of the lining of the digestive tract), alopecia (hair loss), organ dysfunction, and even 
anemia or thrombocytopenia. These side effects sometimes impose dose reduction, treatment delay, or discontinuance of the given therapy [62]. Furthermore, chemotherapeutic agents often cannot penetrate and reach the core of solid tumors, failing to kill the cancerous cells [63].

Traditional chemotherapeutic agents often get washed out from the circulation being engulfed by macrophages. Thus they remain in the circulation for a very short time and cannot interact with the cancerous cells making the chemotherapy completely ineffective. The poor solubility of the drugs is also a major problem in conventional chemotherapy making them unable to penetrate the biological membranes [64]. Another problem is associated with P-glycoprotein, a multidrug resistance protein that is overexpressed on the surface of the cancerous cells, which prevents drug accumulation inside the tumor, acting as the efflux pump, and often mediates the development of resistance to anticancer drugs. Thus the administered drugs remain unsuccessful or cannot bring the desired output $[60,65]$.

\subsection{Nanorobots in Cancer Treatment}

Cancer could be defined as the group of diseases characterized by the uncontrolled growth and spread of abnormal cells in the body is what defines cancer, and the number of individuals affected each year continues to climb [66]. Cancer takes the first place in the research due to its effect of human life and its cost to the economy. Conforming to the Global Oncology Trend Report, by the IMS Institute for Healthcare Informatics, global spending on cancer medications has reached to $\$ 100$ billion in 2014 [4].

Cancer treatment is probably the main reason for the development of nanorobotics, it can be successfully treated with current stages of medical technologies and therapy tools with the help of the nanorobotics. For determine the prognosis and chances for a patient with cancer to survive it could be consider: time of disease evolution considering the diagnosis time if earlier have better prognosis; another important aspect to achieve a successful treatment for patients is the development of efficiently targeted drug delivery to decrease the side effects of chemotherapy [67].

Considering the properties of nanorobots to navigate as bloodborne devices, they can help important treatment processes of complex diseases in early diagnosis and smart drug delivery [41]. A nanorobot can provide an efficient early diagnosis of cancer and help with smart chemotherapy for drug delivery. Nanorobots as drug carriers for timely dosage regimens allows maintaining the chemical compounds for a longer time as necessary into the bloodstream circulation, providing predicted pharmacokinetic parameters for chemotherapy in anti-cancer treatments [21, 68-70].

It avoids the current resulting extravasations towards nonreticuloendothelial-located cancers with the high degenerative side-effects during the chemotherapeutic process [71]. Nanorobots with chemical nanobiosensors can be programmed to detect different levels of E-cadherin and beta-catenin as medical targets in primary and metastatic phases [72, 73], helping target identification and drug delivery.

\subsection{Drug Delivery and Nanorobots in Cancer Treatment}

The clinical use of nanorobots for diagnosis, therapeutic and surgical purposes should be done with intravenous injection. Therefore, the nanorobots can be released directly into the patient's bloodstream. The major cancer treatment cycle for chemotherapy pharmacokinetics includes absorption and metabolism, plus a break for the body's re-establishment before the next chemotherapy session. Patients are normally treated in cycles of every 2 weeks for small tumors [74]. As an initial time threshold for medical purpose, nanorobots should be able to analyze and provide a body diagnosis within one week through the use of proteomic-based sensors. The uptake kinetics of a low molecular weight using a magnetic resonance contrast 
agent can predict the delivery of protein drugs to solid tumors [75]. Hence, a similar approach is useful to verify in vivo nanorobot's biosensor activation through targeted antigen detection [76].

The test and diagnosis is an important part of the research on nanorobotics. It enables rapid testing diagnosis at first visit so without the need of follow-up visit after the lab test, and detection of diseases at an earlier stage. The limitation in in vivo use of nanorobot is the need of energy for propulsion. Higher levels of energy are required since "low inertia and high viscous forces are coupled with low efficiency and low convective motion" [77].

The fuels of chemically powered nanomotors were toxic. The availability of alternative sources of energy such as sound waves [78] and light has led an increase in the research of in vivo use of nanorobots where resulted in more patent applications.

One study of nanomotors is the "Acoustic Propulsion of Nanorod Motors Inside Living Cells" [78] which was a result of the development of ultrasonic-wave powered minerals, which are safe for living systems.

Gao et al. [79] reported an in vivo model of artificial micromotors in a living organism. The model examines the distribution, retention, cargo delivery, and acute toxicity role of synthetic motors in mouse stomach via oral administration. This work is anticipated to significantly advance the emerging field of nano/micromotors and to open the door to in vivo evaluation and clinical applications of these synthetic motors". This development may be an important step for the possibility of in vivo applications of the drug delivery for the cancer treatment with decreasing the side effects of chemotherapy. Juul et al. [80] published a paper on their research into nanorobots that contain medicine that can be opened and closed based on the surrounding temperatures.

Recently, Park et al. (2014) [81] reported a bacteria-based microrobot (bacteriobot) as a new type of active drug delivery system. In the study, genetically modified non-toxic Salmonella typhi-murium (flagellar bacteria) which is attracted to chemicals released by cancer cells are used.

Perrault and Shih from Wyss Institute for Biologically Inspired Engineering at Harvard University introduced virus-inspired enveloped DNA nanostructures as a design strategy for biomedical applications [82].

Recent studies revealed that nanotechnology, DNA engineering of molecular-scale devices with superb control over geometry and site-specific functionalization promises fascinating advantages in advancing nanomedicine. However; instability in biological environments and innate immune activation remain as obstacles for in vivo application.

After nanorobots cross cellular membranes for targeted delivery, drug retention in the tumor will determine the therapeutic efficiency. The chemotherapy is influenced by drug transfer processes from plasma to tissue in achieving more effective tumor chemotherapy based on its composition [75]. Thus, the major advantage of nanorobots for cancer drug delivery is to minimize chemotherapy side effects. As the best approach, the nanorobot architecture incorporates CNT (Carbon nanotubes) and DNA (Deoxyribonucleic acid) that are recent candidates for new forms of nanoelectronics [83].

A CMOS (Complementary metal oxide semiconductor) for constructing circuits with feature sizes in the tens of nanometers [84] as a hybrid biosensor with single-chain antigen-binding proteins $[85,86]$. This process uses activation based on proteomics and bioelectronics signals for medicament release. Therefore, each time the nanorobot detects predefined changes of protein gradients, nanoactuators are activated to manipulate drug delivery.

Changes to chemical and thermal signals are applicable conditions directly related to major medical target identification. Some examples of 
changing protein concentrations inside the body near a medical target under pathological circumstances are NOS (Nitric oxide synthase) [87], E-cadherin [72] and Bcl-2 [88]. Moreover, a change of temperature normally occurs for inflamed tissues [89]. The model incorporates chemical and thermal parameters as clinically and therapeutically the most important guidelines on nanorobot prototyping analysis. The simulation in a $3 \mathrm{D}$ real-time environment aims to establish a useful framework as a testbed for nanorobot foraging inside the body. One of the advances shows a hardware architecture based on Nanobioelectronics is described for the application of nanorobots for cancer therapy $[90,91]$.

The scientists have genetically modified salmonella bacteria that are drawn to tumors by chemicals secreted by cancers cells. The bacteria carry microscopic robots, about 3 micrometers in size that automatically release capsules filled with drugs when the bacteria reach the tumor. By delivering drugs directly to the tumor, the nanorobot, which the team named bacteriobot, attacks the tumor while leaving healthy cells alone, sparing the patient from the side effects of chemotherapy. Bactiriobot can only detect tumor-forming cancers, such as breast cancers and colorectal, but the nanorobot will eventually be able to treat other cancers as well [75]. Another advance of nanorobots and drug delivery has avoided by using existing organisms such the 'magnetotactic bacteria' Magnetospirillum gryphiswaldense. These bacteria have a natural magnetic polarity that allows them to be moved by computational external electrical currents combined to form magnetotaxis [92].

Faced with the bottlenecks found in those studies and the existence of other more, you can observe this phenomenon in the survey conducted in database Web Of Science [49], about "Drug delivery and Cancer", Drug delivery and Nanorobotics" and Drug delivery and Treatment of cancer". The results are depicted in Fig. 1.

The results of Fig. 1 refers to the number of publications already made and that are present in databases available by Web of Science portal. Despite current technological advances and application prospects of this impressive nanotechnology, these results indicated the complexity of the issue and the

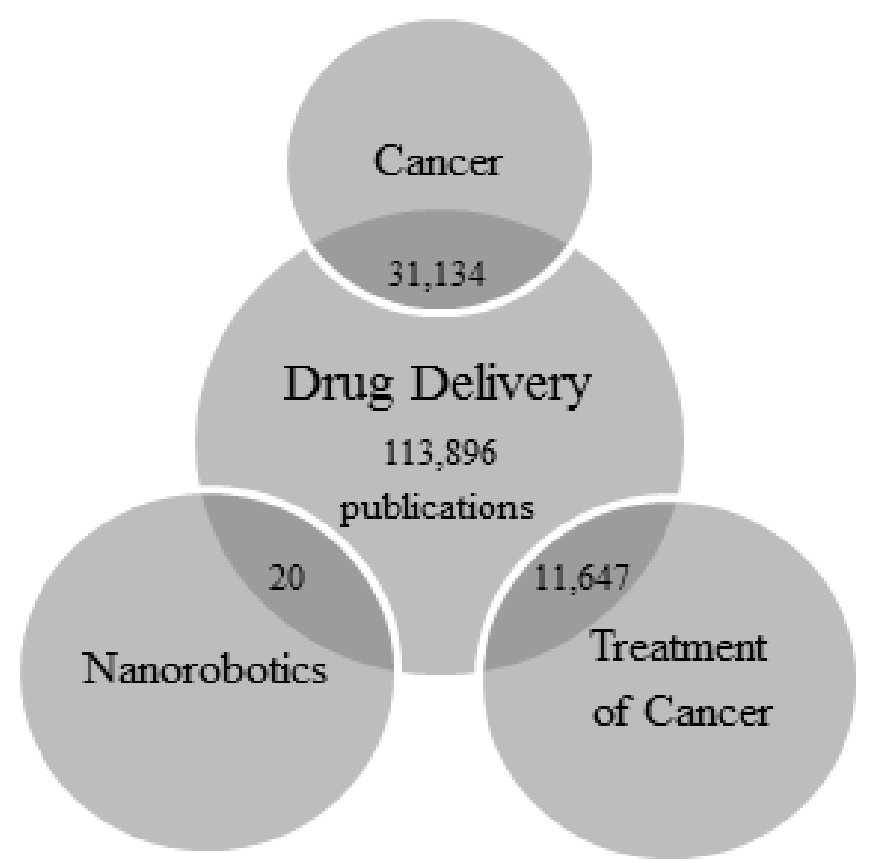

Fig. 1 Venn's Diagram showing the number of works already published on the topics: “drug delivery”, “drug delivery and cancer”, “drug delivery and treatment of cancer” and “drug delivery and nanorobotics”, according Web of Science Database [49]. 
difficulty of achieving tangible results. Also demonstrates the need for greater investment to conduct research in the area. However, it is observed that the evolution of nanotechnology possibly triggers an accelerated development of all research related to nanoworld, including rapid insertion of nanorobots in conventional medicine.

\section{Conclusions}

This review aimed at giving an overview of the present status of nanorobotics in cancer therapy. Cancer takes the first place in the research due to its effect of human life and its cost to the economy. So, it was presented a study on different approaches employed towards cancer treatment using nanorobots. Thereby, this review focused on recent advances and technology development of nanorobotics harvesting specifically for Drug Delivery Systems application for treatment of cancer. Therefore, cancer treatment is probably the main reason for the development of nanorobotics, i.e., it can be successfully treated with current stages of medical technologies and therapy tools with the help of the nanorobotics.

\section{Acknowledgments}

The authors wish to thank to Coordenação de Aperfeiçoamento de Pessoal de Nível Superior (CAPES) for financial support. Thanks to Nanotecnology Laboratory (NANOTEC-FGA/UnB) at University of "' Brasília by technical support and installations.

\section{References}

[1] Barbosa, G., Silva, P. A. F., Luz, G. V. S. and Brasil, L. M. 2015. "Nanotechnology Applied in Drug Delivery." In World Congress on Medical Physics and Biomedical Engineering 51st Edition, Toronto, Canadá: Springer International Publishing Switzerland 911-14.

[2] Abhilash, M. 2010. "Nanorobots." International Journal of Pharma and Bio Sciences V1 (1): 1-10.

[3] Vettiger, P., Cross, G., Despont, M., Drechsler, Dürig, U., Gotsmann, B., Häberle W., Lantz, M. A., Rothuizen, H. E., Stutz, R. and Binnig, G. K. 2002. "The "Millipede" -
Nanotechnology Entering Data Storage.” IEEE Transactions on Nanotechnology 1 (1): 39-55.

[4] Developments in Cancer Treatments, Market Dynamics, Patient Access and Value. Accessed May 31, 2016. http://www.imshealth.com/en/thought-leadership/ims-inst itute/reports/global-oncology-trend-2015.

[5] Ross, J. S., Schenkein, D. P., Pietrusko, R., Rolfe, M., Linette, G. P., Stec, J., Stagliano, N. E., Ginsburg, G. S., Symmans, W. F., Pusztai, L. and Hortobagyi, G. N. 2004. "Targeted Therapies for Cancer 2004." American Journal of Clinical Pathology 122 (4): 598-609.

[6] Maeda, H. 2001. "The Enhanced Permeability and Retention (EPR) Effect in Tumor Vasculature: The Key Role of Tumor-Selective Macromolecular Drug Targeting." Advances in Enzyme Regulation 41: 189-207.

[7] Iglessias, A. S., de Moraes, A. J. V., Marion, B. D. G., Ferreira, L. S. and Hasan, N. M. 2014. "The Posibilities of Nanotechnology." In World Congress on Medical Physics and Biomedical Engineering (COBENGE), 10.

[8] Invernizzi, N. 2007. Future Vision: Nanoscience and Nanotechnology. Brasilian Society for Science Progress (SBPC).

[9] Medina-Sánchez, M., Schwarz, L., Meyer, A. K., Hebenstreit, F. and Schmidt, O. G. 2016. "Cellular Cargo Delivery: Toward Assisted Fertilization by Sperm-Carrying Micromotors." Nano Letters 16 (1): 555-61.

[10] Wang, J. 2009. "Can Man-Made Nanomachines Compete with Nature Biomotors?” ACS Nano 3 (1) 4-9.

[11] Uygun, M., Singh, V. V, Kaufmann, K., Uygun, D. A., de Oliveira, S. D. S., and Wang, J. 2015. "Micromotor-Based Biomimetic Carbon Dioxide Sequestration: Towards Mobile Microscrubbers." Angewandte Chemie (International ed. in English) 54 (44) : 12900-4.

[12] Freitas, R. A. 2005. "What is Nanomedicine?" Nanomedicine: Nanotechnology, Biology and Medicine 1 (1): 2-9.

[13] Coluzza, I., van Oostrum, P. D. J., Capone, B., Reimhult, E. and Dellago, C. 2013. "Sequence Controlled Self-Knotting Colloidal Patchy Polymers." Physical Review Letters 110 (7) : 075501.

[14] A Brazilian Agency Of Industrialdevelopment. 2010. "Nanotechnology Prospective Study." Journal of Chemical Information and Modeling 53.

[15] Mallouk, T. E. and Sen, A. 2009. "Powering Nanorobots." Scientific American 300 (5): 72-7.

[16] Neal, R. W. 2014. Cancer-Fighting Robot: Korean Scientists Develop Nanorobots That Are More Efficient Than Chemotherapy. International Business Times 
Reuters, U.S. Accessed May 31, 2016. http://www.ibtimes.com/cancer-fighting-robot-korean-sci entists-develop-nanorobots-are-more-efficient-chemother apy-video.

[17] Ronconi, C. M. 2011. "Nanomachines: Chemists as the Architect of the World." Science Today 48: 34-9.

[18] Palagi, S., Mark, A. G., Reigh, S. Y., Melde, K., Qiu, T., Zeng, H., Parmeggiani, C., Martella, D., Sanchez-Castillo, A., Kapernaum, N., Giesselmann, F., Wiersma, D. S., Lauga, E. and Fischer, P. 2016. "Structured Light Enables Biomimetic Swimming and Versatile Locomotion of Photoresponsive Soft Microrobots." Nature Materials 15 (6): 647-53.

[19] Chowdhury, S., Jing, W. and Cappelleri, D. J. 2016. "Towards Independent Control of Multiple Magnetic Mobile Microrobots.” Micromachines 7 (1): 1-14.

[20] Barrier, B. 2014. "Guidelines for the Design of Magnetic Nanorobots." 30 (1): 81-92.

[21] Bhat, A. S. 2014. "Nanobots: The Future of Medicine." International Journal of Engineering and Management Sciences 5 (1): 44-9.

[22] Ricotti, L. and Menciassi, A. 2015. "Nanotechnology in Biorobotics: Opportunities and challenges." Journal of Nanoparticle Research 17 (2): 1-10.

[23] Lenaghan, S. C., Wang, Y., Xi, N., Fukuda, T., Tarn, T., Hamel, W. R. and Zhang, M. 2013. "Grand Challenges in Bioengineered Nanorobotics for Cancer Therapy." IEEE Transactions on Biomedical Engineering 60 (3): 667-73.

[24] Davis, M. E., Chen, Z. G. and Shin, D. M. 2008. "Nanoparticle Therapeutics: An Emerging Treatment Modality for Cancer." Nature Reviews. Drug Discovery 7 (9): 771-82.

[25] Cho, K., Wang, X., Nie, S., Chen, Z. and Shin, D. M. 2008. "Therapeutic Nanoparticles for Drug Delivery in Cancer." Clinical Cancer Research 14 (5): 1310-6.

[26] Glass, P., Cheung, E., Hanyan Wang, R., Appasamy, M. and Sitti, M. 2008. "A Motorized Anchoring Mechanism for a Tethered Capsule Robot Using Fibrillar Adhesives for Interventions in the Esophagus." In 2008 2nd IEEE RAS \& EMBS International Conference on Biomedical Robotics and Biomechatronics. 758-64.

[27] Jia, X., Li, X., Lenaghan, S. C. and Zhang, M. 2014. "Design of Efficient Propulsion for Nanorobots." IEEE Transactions on Robotics 30 (4): 792-801.

[28] Lenaghan, S. C., Chen, J. and Zhang, M. 2013. "Modeling and Analysis of Propulsion in the Multiflagellated Micoorganism Giardia Lamblia." Physical Review E - Statistical, Nonlinear, and Soft Matter Physics 88 (1): 1-9.

[29] Young, L. H., Hyun, L. N., Gilson, A, S. J., Jungahn, K., Bang, L. H. and Hang, C. S. 2005. "Preparation of
Poly(vinylpyrrolidone) Coated Iron Oxide Nanoparticles for Contrast Agent." Polymer Korea 29 (3): 266-70.

[30] Liu, H.-L., Ko, S. P., Wu, J.-H., Jung, M.-H., Min, J. H., Lee, J. H., An, B. H. and Kim, Y. K. 2007. "One-Pot Polyol Synthesis of Monosize PVP-Coated Sub-5nm $\mathrm{Fe}_{3} \mathrm{O}_{4}$ Nanoparticles for Biomedical Applications." Journal of Magnetism and Magnetic Materials 310 (2): e815-7.

[31] Yu, D.-H., Liu, Y.-R., Luan, X., Liu, H.-J., Gao, Y.-G., Wu, H., Fang, C. and Chen, H.-Z. 2015. "IF7-Conjugated Nanoparticles Target Annexin 1 of Tumor Vasculature against P-gp Mediated Multidrug Resistance." Bioconjugate Chemistry 26 (8): 1702-12.

[32] Pharma, E. 2016. EyeGate II Delivery System. Accessed June $\quad 04,2016$. http://www.eyegatepharma.com/technology/eyegate-ii-de livery-system/.

[33] Johnson, D. 2014. Graphene Transforms Itself Into a Sphere for Drug Delivery - IEEE Spectrum. IEEE Spectrum. Accessed April 07, 2016. http://spectrum.ieee.org/nanoclast/biomedical/devices/gra phene-transforms-itself-into-a-sphere-opening-up-medica 1-applications.

[34] Vartholomeos, P., Fruchard, M., Ferreira, A. and Mavroidis, C. 2011. "MRI- Guided Nanorobotic Systems for Therapeutic and Diagnostic Applications." In Annual Review of Biomedical Engineering 13: 157-84.

[35] Ungaro, F., d'Angelo, I., Miro, A., La Rotonda, M. I. and Quaglia, F. 2012. "Engineered PLGA Nano- and Micro-Carriers for Pulmonary Delivery: Challenges and Promises." The Journal of Pharmacy and Pharmacology 64 (9): 1217-35.

[36] Pappu, P., Madduru, D., Chandrasekharan, M., Modhukur, V., Nallapeta, S. and Suravajhala, P. 2016. "Next Generation Sequencing Analysis of Lung Cancer Datasets: A functional Genomics Perspective." Indian Journal of Cancer 53 (1): 1-8.

[37] Verma, S. K. and Chauhan, R. 2014. "Nanorobotics in Dentistry - A review." Indian Journal of Dentistry 5: 62-70.

[38] Dixon, K. L. 2003. "The Radiation Biology of Radioimmunotherapy." Nuclear Medicine Communications 24 (9) : 951-57.

[39] Hussan Reza, K., Asiwarya, G., Radhika, G. and Bardalai, D. 2011. "Nanorobots: The future Trend of Drug Delivery and Therapeutics." International Journal of Pharmaceutical Sciences Review and Research 10 (1): 60-8.

[40] Robert A. and 2011. "Medical Nanorobotics: The Long-Term Goal for Nanomedicine." 367-92.

[41] Freitas, R. A. 2006. "Pharmacytes: An Ideal Vehicle for 
Targeted Drug Delivery." Journal of Nanoscience and Nanotechnology 6 (9-10): 2769-75.

[42] Freitas, R. a 2005. "Microbivores: Artificial Mechanical Phagocytes Using Digest and Discharge Protocol." Journal of Evolution and Technology 14 (April): 1-45.

[43] Manjunath, A. and Kishore, V. 2014. "The Promising Future in Medicine: Nanorobots." Biomedical Science and Engineering 2 (2): 42-7.

[44] Karan, S., Banerjee, B., Tripathi, A. and Majumder, D. D. 2015. "Nanorobotics Control Systems Design - A New Paradigm for Healthcare System - Bookmetrix Analysis." In Emerging ICT For Bridging The Future - Proceedings Of The 49th Annual Convention Of The Computer Society Of India (CSI), Volume 1.

[45] Bhowmik, D., Chiranjib, B. and Jayakar, R. M. C. 2009. "Role of Nanotechnology in Novel Drug Delivery System." Journal of Pharmaceutical Science And Technology 1 (1): 20-35.

[46] Robert, B. and Jr, A. F. IMM Report Number 18: Nanomedicine Clottocytes: Artificial Mechanical Platelets. 2016.

[47] Dabbs, D. J. 2014. Diagnostic Immunohistochemistry: Theranostic and Genomic Applications 4th edition. Philadelphia: ELSEVIER SAUNDERS.

[48] Portal periodicos CAPES. Accessed May 31, 2016. http://www.periodicos.capes.gov.br/?\&pds_handle $=3052$ $016162650163114601846082594148 \&$ calling_system $=p r$ imo\&institute $=$.

[49] Web of Science [v.5.22] - All databases. Accessed June 01 , 2016. http://apps.webofknowledge.com/UA_GeneralSearch_inp ut.do? product $=$ UA\&search $\_$mode $=$GeneralSearch\&SID $=$ 3DCzfvN7XkKHGA5i9Hu\&preferencesSaved=.

[50] Golan, D. E., Tashjian Jr., A. H., Armstrong, E. J. and Armstrong, A. W. 2011. Principles of Pharmacology: The Pathophysiologic Basis of Drug Therapy. LWW; 3rd edition (June 24, 2011), 976.

[51] Rang, H. P., Ritter, J. M., Flower, R. J. and Henderson, G. 2015. Rang \& Dale's Pharmacology 8th Editio. Churchill Livingstone, 776.

[52] Brasil Acessed 2015, Publicadas novas normas para pesquisa clínica., Agência Nacional de Vigilância Sanitária-Anvisa. Accessed May 24, 2016. $\mathrm{http} / /$ portal.anvisa.gov.br/wps/content/anvisa+portal/anvi sa/sala+de+imprensa/menu+-+noticias+anos/2015/public adas + novas + normas + para + pesquisa + clinica.

[53] Kratz, F. and Warnecke, A. 2012. "Finding the Optimal Balance: Challenges of Improving Conventional Cancer Chemotherapy Using Suitable Combinations with Nano-Sized Drug Delivery Systems." J Control Release 164: 221-35.

[54] Zeeshan, M. A., Pané, S., Youn, S. K., Pellicer, E.,
Schuerle, S., Sort, J., Fusco, S., Lindo, A. M., Park, H. G. and Nelson, B. J. 2013. "Graphite Coating of Iron Nanowires for Nanorobotic Applications: Synthesis, Characterization and Magnetic Wireless Manipulation." Advanced Functional Materials 23 (7): 823-31.

[55] Kojima, C., Suehiro, T., Watanabe, K., Ogawa, M., Fukuhara, A., Nishisaka, E., Harada, A., Kono, K., Inui, T. and Magata, Y. 2013. "Doxorubicin-Conjugated Dendrimer/Collagen Hybrid Gels for Metastasis-Associated Drug Delivery Systems." Acta Biomaterialia 9 (3): 5673-80.

[56] Scialabba, C., Licciardi, M., Mauro, N., Rocco, F., Ceruti, M. and Giammona, G. 2014. "Inulin-Based Polymer Coated SPIONs as Potential Drug Delivery Systems for Targeted Cancer Therapy." European Journal of Pharmaceutics and Biopharmaceutics: Official Journal of Arbeitsgemeinschaft Für Pharmazeutische Verfahrenstechnik e.V 88 (3): 695-705.

[57] Watanabe, K., Nishio, Y., Makiura, R., Nakahira, A. and Kojima, C. 2013. "Paclitaxel-Loaded Hydroxyapatite/Collagen Hybrid Gels as Drug Delivery Systems for Metastatic Cancer Cells.” International Journal of Pharmaceutics 446 (1-2) : 81-6.

[58] Liu, Z., Robinson, J. T., Tabakman, S. M., Yang, K. and Dai, H. 2011. "Carbon Materials for Drug Delivery \& Cancer Therapy." Materials Today 14 (7-8): 316-23.

[59] Health Quality Ontario 2016. Intrathecal Drug Delivery Systems for Cancer Pain: A Health Technology Assessment. 16 (1): 1-51.

[60] Sutradhar, K. B. and Amin, M. L. 2014. "Nanotechnology in Cancer Drug Delivery and Selective Targeting." ISRN Nanotechnology 2014. 12.

[61] Zhao, G. and Rodriguez, B. L. 2013. "Molecular Targeting of Liposomal Nanoparticles to Tumor Microenvironment." International Journal of Nanomedicine 8: 61-71.

[62] Coates, A., Abraham, S., Kaye, S. B., Sowerbutts, T., Frewin, C., Fox, R. M. and Tattersall, M. H. 1983. "On the Receiving End--Patient Perception of the Side-Effects of Cancer Chemotherapy." European Journal of Cancer \& Clinical Oncology 19 (2): 203-8.

[63] Tannock, I. F., Lee, C. M., Tunggal, J. K., Cowan, D. S. M. and Egorin, M. J. 2002. "Limited Penetration of Anticancer Drugs Through Tumor Tissue: A Potential Cause of Resistance of Solid Tumors to Chemotherapy." Clinical Cancer Research: An Official Journal of the American Association for Cancer Research 8 (3): 878-84.

[64] Mousa, S. A. and Bharali, D. J. 2011. "Nanotechnology-Based Detection and Targeted Therapy in Cancer: Nano-Bio Paradigms and Applications." Cancers 3 (3): 2888-903. 
[65] Brown, R. and Links, M. 2004. "Clinical Relevance of the Molecular Mechanisms of Resistance to Anti-Cancer Drugs." Expert Reviews in Molecular Medicine 1 (15): $1-21$.

[66] World Health Organization Acessed 2015, Cancer. Accessed May 31, 2016. http://www.who.int/cancer/en/.

[67] Kshirsagar, N., Patil, S., Kshirsagar, R., Wagh, A. and Bade, A. 2014. "Review on Application of Nanorobots in Health Care." World Journal of Pharmacy and Pharmaceutical Sciences 3 (5): 472-80.

[68] Mutoh, K., Tsukahara, S., Mitsuhashi, J., Katayama, K. and Sugimoto, Y. 2006. "Estrogen-Mediated Post Transcriptional Down-Regulation of P-Glycoprotein in MDR1-Transduced Human Breast Cancer Cells." Cancer Science 97 (11): 1198-204.

[69] Lagzi, I. 2013. "Chemical Robotics - Chemotactic Drug Carriers." Open Medicine 8 (4): 377-82.

[70] Xu, X., Kim, K. and Fan, D. 2015. "Tunable Release of Multiplex Biochemicals by Plasmonically Active Rotary Nanomotors." Angewandte Chemie (International ed. in English) 54 (8): 2525-9.

[71] Couvreur, P. 2006. "Nanotechnologies for Drug Delivery: Application to Cancer and Autoimmune Diseases." Progress in Solid State Chemistr 34 (2): 231-5.

[72] Janda, E., Nevolo, M., Lehmann, K., Downward, J., Beug, H. and Grieco, M. 2006. "Raf Plus TGFBeta-Dependent EMT is Initiated by Endocytosis and Lysosomal Degradation of E-Cadherin." Oncogene 25 (54): 7117-30.

[73] Who We Are | Sanger Institute. Accessed May 31, 2016. http://www.sanger.ac.uk/about/who-we-are.

[74] Osterlind, K. 2001. "Chemotherapy in Small Cell Lung Cancer.” European Respiratory Journal 18 (6): 1026-43.

[75] Artemov, D., Solaiyappan, M. and Bhujwalla, Z. M. 2001. "Magnetic Resonance Pharmacoangiography to Detect and Predict Chemotherapy Delivery to Solid Tumors." Cancer Research 61 (7): 3039-44.

[76] Cavalcanti, A., Shirinzadeh, B., Freitas Jr, R. A, and Hogg, T. 2007. "Nanorobot Architecture for Medical Target identification." Nanotechnology 19 (1): 015103.

[77] Sharma, N. N. and Mittal, R. K. 2008. "Nanorobot Movement: Challenges and Biologically Inspired Solutions." International Journal on Smart Sensing and Intelligent Systems 1 (1): 87-109.

[78] Wang, W., Li, S., Mair, L., Ahmed, S., Huang, T. J. and Mallouk, T. E. 2014. "Acoustic Propulsion of Nanorod Motors Inside Living Cells.” Angewandte Chemie 126 (12): 3265-68.

[79] Gao, W., Dong, R., Thamphiwatana, S., Li, J., Gao, W., Zhang, L. and Wang, J. 2015. "Artificial Micromotors in the Mouse's Stomach: A Step Toward in vivo Use of Synthetic Motors." ACS Nano 9 (1): 117-23.
[80] Juul, S., Iacovelli, F., Falconi, M., Kragh, S. L., Christensen, B., Frøhlich, R., Franch, O., Kristoffersen, E. L., Stougaard, M., Leong, K. W., Ho, Y.-P., Sørensen, E. S., Birkedal, V., Desideri, A. and Knudsen, B. 2013. "Temperature-Controlled Encapsulation and Release of an Active Enzyme in the Cavity of a Self-Assembled DNA Nanocage." ACS nano 7 (11): 9724-34.

[81] Park, D., Park, S. J., Cho, S., Lee, Y., Lee, Y. K., Min, J.-J., Park, B. J., Ko, S. Y., Park, J.-O. and Park, S. 2014. "Motility Analysis of Bacteria-Based Microrobot (Bacteriobot) Using Chemical Gradient Microchamber." Biotechnology and Bioengineering 111 (1): 134-43.

[82] Perrault, S. D. and Shih, W. M. 2014. "Virus-Inspired Membrane Encapsulation of DNA Nanostructures to Achieve in vivo Stability." ACS Nano 8 (5): 5132-40.

[83] Li, H., Carter, J. D. and LaBean, T. H. 2009. "Nanofabrication by DNA Self-Assembly." Materials Today 12 (5): 24-32.

[84] Goldstein, S. C. and Rosewater, D. L. 2006. Methods of chemically assembled electronic nanotechnology circuit fabrication. US Patent 7,064,000, filed July, and issued June 20, 2004.

[85] Im, H., Huang, X.-J., Gu, B. and Choi, Y.-K. 2007. “A Dielectric-Modulated Field-Effect Transistor for Biosensing." Nature Nanotechnology 2 (7): 430-4.

[86] Pison, U., Giersig, M. and Schäfer, A. 2011. Diagnostic Nanosensor and Its Use in Medicine. GILUPI GmbH Patent EP 1811302 B1, filed January 19, 2006, and issued August 10, 2011.

[87] Fukuda, S., Hashimoto, N., Naritomi, H., Nagata, I., Nozaki, K., Kondo, S., Kurino, M. and Kikuchi, H. 2000. "Prevention of Rat Cerebral Aneurysm Formation by Inhibition of Nitric Oxide Synthase." Circulation 101 (21): 2532-8.

[88] Callagy, G. M., Pharoah, P. D., Pinder, S. E., Hsu, F. D., Nielsen, T. O., Ragaz, J., Ellis, I. O., Huntsman, D. and Caldas, C. 2006. "Bcl-2 is a Prognostic Marker in Breast Cancer Independently of the Nottingham Prognostic Index." Clinical Cancer Research: An Official Journal of the American Association for Cancer Research 12 (8): 2468-75.

[89] Tan, T. Z., Quek, C., Ng, G. S. and Ng, E. Y. K. 2007. “A Novel Cognitive Interpretation of Breast Cancer Thermography with Complementary Learning Fuzzy Neural Memory Structure." Expert Systems with Applications 33 (3): 652-66.

[90] Sivasankar, M. and Durairaj, R. 2012. "Brief Review on Nano Robots in Bio Medical Applications." Adv Robot Autom. 1: 101.

[91] Karan, S., Dutta Majumder, D. and Chaudhuri, S. 2012. Biological Response Modifier - A Nanorobotics Control System Design for Immunotherapy in Cancer Treatment. 
In 2012 1st International Conference on Recent Advances in Information Technology (RAIT). 645-50.

[92] Martel, S., Tremblay, C. C., Ngakeng, S. and Langlois,
G. 2006. "Controlled Manipulation and Actuation of Micro-Objects with Magnetotactic Bacteria." Applied Physics Letters 89 (23): 9-11. 